Towar ds cross- hi er ar chy si mil at i on of col I i si onl ess dri ven reconnect i on i $n$ an open syst em

\begin{tabular}{|l|l|}
\hline $\begin{array}{l}\text { j our nal or } \\
\text { publ i cat i on } \mathrm{ti} \text { t l e }\end{array}$ & Journal of Pl asna Physi cs \\
\hline vol une & Vol . 72 \\
\hline nunber & I ssue 6 \\
\hline page range & pp. 953 956 \\
\hline year & 2006-12-01 \\
\hline URL & ht t p: //hdl . handl e. net $/ 10655 / 1298$ \\
\hline
\end{tabular}




\title{
Towards cross-hierarchy simulation of collisionless driven reconnection in an open system
}

\author{
R. H OR I UCHI I,2, H. OH T A NI I,2 and A. I S H I Z A W A ${ }^{1}$ \\ ${ }^{1}$ National Institute for Fusion Science, Oroshi-cho 322-6, Toki 509-5292, Japan \\ ${ }^{2}$ The Graduate University for Advanced Studies (Soken-dai), Oroshi-cho 322-6, \\ Toki 509-5292, Japan
}

(Received 30 August 2005 and accepted 13 June 2006)

\begin{abstract}
The basic idea of a cross-hierarchy model for magnetic reconnection in an open system is proposed, where a microscopic system is surrounded by a macroscopic system and the interaction between the two systems is expressed by the plasma inflow and outflow through the system boundary. Collisionless driven reconnection in two-dimensional and three-dimensional open systems is demonstrated using an open particle simulation model developed as a microscopic part of a cross-hierarchy model. It is found that the openness of the system and scalecoupling effects play crucial roles in collisionless driven reconnection.
\end{abstract}

\section{Introduction}

Magnetic reconnection is controlled by multi-scale physics from microscopic physics, which leads to the generation of electric resistivity, through macroscopic physics, which determines global plasma transport and global change of field topology. In other words, it functions as the bridging process between the macroscopic world and microscopic world. As these physics are closely coupled with each other, a full understanding of magnetic reconnection requires a cross-hierarchy model which can treat both microscopic physics and macroscopic physics simultaneously and solve their scale-coupling consistently.

In this paper we propose the following basic idea of a cross-hierarchy model based on the domain division method. A microscopic system in which microscopic physics plays a crucial role exists in the vicinity of the reconnection point. A macroscopic system in which magnetic field is frozen in plasmas and so physical processes associated with magneto-fluid motion are dominantly important exists in the surroundings of the microscopic system. Dynamics in the microscopic system are described by electromagnetic particle simulation, while macroscopic physics are described by magnetohydradynamic (MHD) simulation. The interaction between two systems is expressed by the plasma inflow and outflow through the system boundary. As a first step for cross-hierarchy simulation of magnetic reconnection, we have developed an electromagnetic particle simulation model for magnetic reconnection in an open system ('PASMO') responsible for the microscopic part of a crosshierarchy model, in which the information of macroscopic system is introduced in the form of boundary conditions [1-3]. 

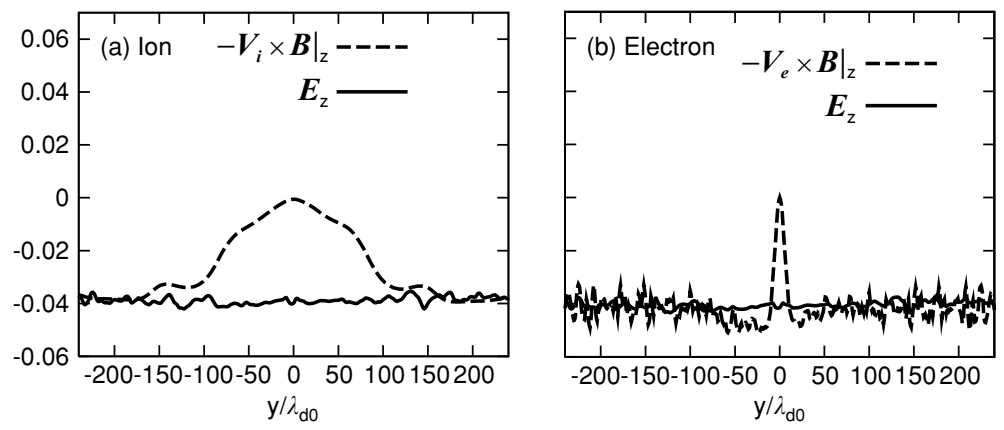

Figure 1. Spatial profiles of frozen-in condition for (a) ions and (b) electrons.

\section{Open boundary model for particle simulation}

Let us consider the dynamical evolution of a microscopic system with a fixed rectangular domain which is subject to an external driving source. Simulation code relies on the explicit electromagnetic particle-in-cell method [4]. The floating condition is used at the downstream boundary $\left(x= \pm x_{\mathrm{b}}\right)$. Field quantities are assumed to be continuous at the downstream boundary. The boundary condition for particles is determined so that charge neutrality is maintained and the net number flux of particles at the boundary is equal to that associated with the fluid velocity in the vicinity of the downstream boundary.

The frozen-in condition, $\mathbf{E}+\mathbf{v}_{s} \times \mathbf{B}=0(s=\mathrm{e}, \mathrm{i})$, is assumed at the upstream boundary $\left(y= \pm y_{\mathrm{b}}\right)$ in addition to the conditions as $E_{z}=E_{\mathrm{d}}(x, t)$ and $E_{x}=0$. The other field quantities at the boundaries can be determined completely by solving Maxwell equations. Here, $E_{\mathrm{d}}(x, t)$ is an external driving field, which plays a role in driving the plasma inflow from an external macroscopic region. In this paper the driving field is given by some functional form without connecting two systems to check the validity of this microscopic model [2]. The distribution function of incoming particles at the upstream boundary is assumed to be a shifted Maxwellian with a constant temperature and the average velocity equal to the $\mathbf{E} \times \mathbf{B}$ drift velocity. Figure 1 illustrates the spatial profiles of two quantities in the frozenin condition obtained using developed two-dimensional simulation code ('PASMO2D') for the case of mass ratio $M_{\mathrm{i}} / M_{\mathrm{e}}=800$ and the strength of the driving field $E_{0}=-0.04 B_{0}\left(B_{0}\right.$ is the magnetic field amplitude in an initial equilibrium) [5]. The frozen-in condition is clearly satisfied near the upstream boundary for both electrons and ions during the simulation.

We have examined the dynamical behavior of collisionless driven reconnection for the driving field with different spatial profiles and amplitudes by making use of PASMO-2D [2]. It is found that there are two regimes in the long time scale behavior of collisionless reconnection which are controlled mainly by the spatial scale of plasma inflow, i.e. steady regime and intermittent regime. In the small spatial scale case the system evolves towards a steady regime in which steady reconnection is realized and thus the global field topology remains unchanged. On the other hand, in the large spatial scale case the system evolves into an intermittent regime in which magnetic islands are frequently excited near the center of the current sheet. This result suggests that the openness of system or the interaction with the macroscopic system plays a crucial role in the dynamical behavior of magnetic reconnection. 


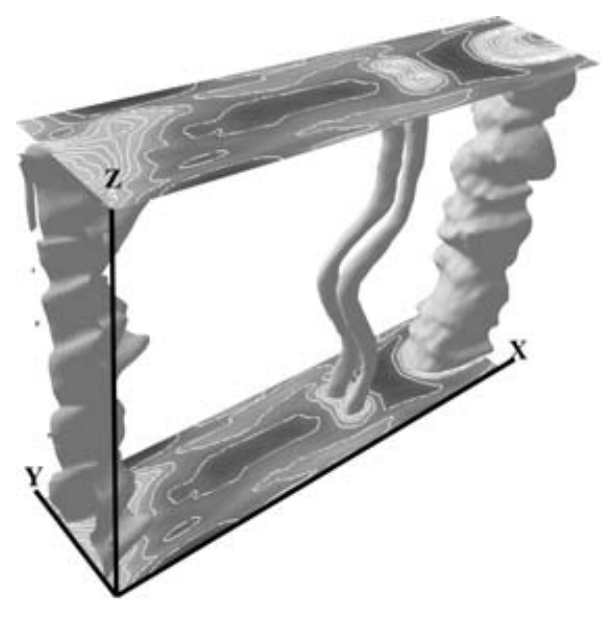

(a)

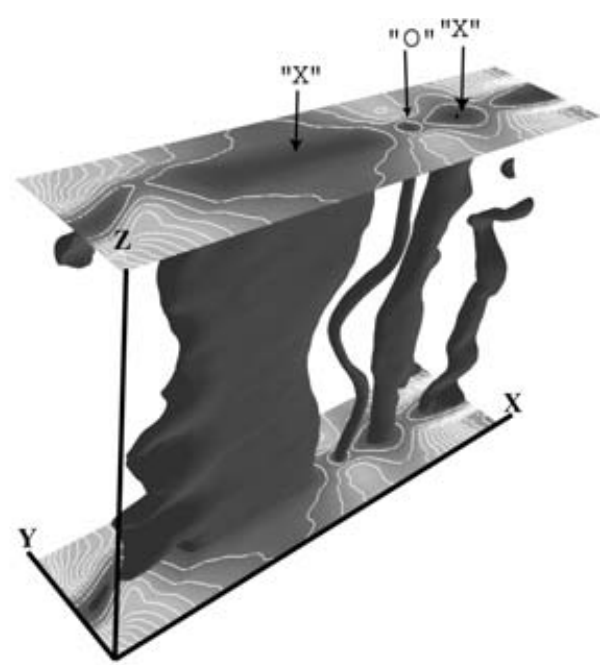

(b)

Figure 2. Perspective views of (a) mass density profile and (b) spatial profile of reconnection component of magnetic field at $t \omega_{\mathrm{ce}}=444$.

\section{Triggering mechanism of collisionless driven reconnection}

Triggering mechanism of collisionless driven reconnection and its dynamical behavior in an open system are investigated by means of three-dimensional full particle simulations based on the developed open boundary model ('PASMO-3D') [3]. The driving electric field imposed at the upstream boundary carries the plasma towards the current sheet and compresses it. The non-ideal effects such as the inertia effect and the meandering effect break the frozen-in condition of magnetic field in the central current region. Once collisionless reconnection is triggered, fast reconnection flow is generated and carries the magnetic flux towards the downstream region. The current sheet is split as a result of collisionless reconnection, and thus small islands appear in the downstream. Figure 2 demonstrates the perspective views of (a) mass density profile and (b) spatial profile of reconnection component of the magnetic field in the island growing phase for the case of $M_{\mathrm{i}} / M_{\mathrm{e}}=100$ and $E_{0}=-0.04 B_{0}$. The dark areas in the right panel stands for the weak field region, and the ' $\mathrm{o}$ ' and ' $\mathrm{x}$ ' points of magnetic field topology are located at the center of the dark regions. A small island with two peaks of mass density is deformed due to the excitation of a kink instability. The two-peak structure is formed by the mass flow supplied along the filed lines from two ' $\mathrm{x}$ '-points on both sides. After the magnetic islands move out though the boundary, the system relaxes into a quasi-steady state in which the energy inflow is balanced with the energy outflow.

A low-frequency electromagnetic instability, which is called a drift-kink instability (DKI) [3, 6], is excited near the central region in this quasi-steady state. Figure 3 shows the spatial profiles of non-ideal terms when the DKI develops fully at the central region of current sheet. Here, the reconnection point is located at the position $y=0$. In order to examine the violation mechanism of the frozen-in condition we expand the physical quantities into the DC and wavy components. It is found that there are two causes of the violation of the frozen-in constraint and that triggered collisionless reconnection. One is anomalous resistivity created by the DKI, and the other is originated by stochastic particle motion near the neutral 


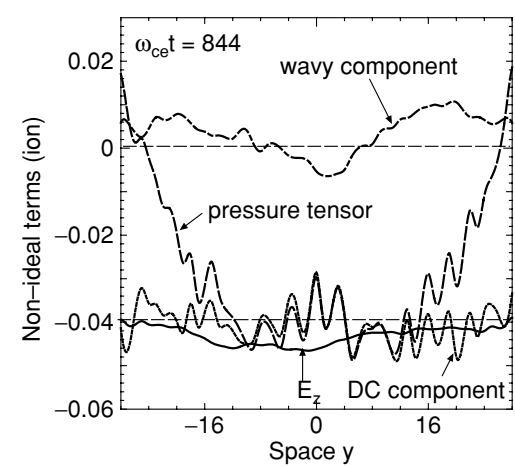

(a)

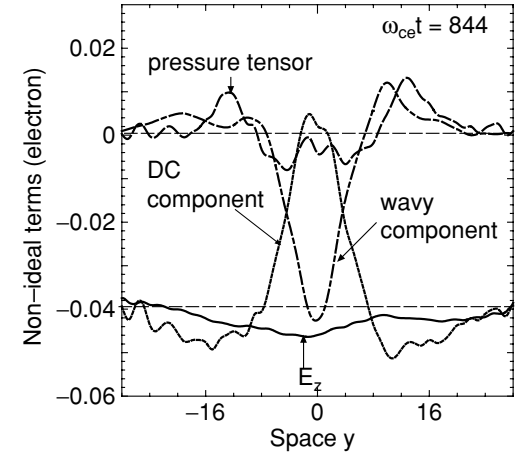

(b)

Figure 3. Spatial profiles of non-ideal terms for (a) ions and (b) electrons.

sheet, which is expressed by off-diagonal components of a pressure tensor term in two-fluid MHD. The reconnection electric field (solid curve) is sustained by the ion pressure tensor originating from stochastic particle orbit (dashed curve) in the ion current layer (Fig. 3(a)). On the other hand, the wavy component (dot-dashed curve) has a strong peak near the reconnection point, and balanced with the reconnection electric field in the electron case (Fig. 3(b)). In other words, anomalous resistivity created by the DKI is a main cause of the reconnection electric field in the electron current layer. Thus, in contrast to the two-dimensional case [5, 7], the global threedimensional structure of current layer affects the growth of the DKI so that it grows just inside an electron current layer.

\section{Summary}

Collisionless driven reconnection in two-dimensional and three-dimensional open systems has been investigated using an open particle simulation model developed as a microscopic part of a cross-hierarchy model. It has been revealed that the openness of system and scale-coupling effects play crucial roles in the dynamical behavior of magnetic reconnection. The relationship between the two causes triggering collisionless driven reconnection, i.e. anomalous resistivity created by the DKI and the effect of stochastic particle motion near the neutral sheet, has been investigated by means of three-dimensional simulations. The macroscopic part of cross-hierarchy model, an open MHD model, is now under development.

\section{References}

[1] Pei, W., Horiuchi, R. and Sato, T. 2001 Phys. Rev. Lett. 87, 235003.

[2] Pei, W., Horiuchi, R. and Sato, T. 2001 Phys. Plasmas 8, 3251-3257.

[3] Horiuchi, R., Ohtani, H. and Ishizawa, A. 2004 Comput. Phys. Commn. 164, 17-22.

[4] Birdsall, C. K. and Langdon, A. B. 1985 In: Plasma Physics via Computer Simulation. New York: McGraw-Hill.

[5] Ishizawa, A. and Horiuchi, R. 2005 Phys. Rev. Lett. 95, 045003.

[6] Zhu, Z. and Winglee, R. M. 1996 J. Geophys. Res. 101, 4885.

[7] Moritaka, T., Horiuchi, R., Ohtani, H. and Ishizawa, A. 2006 J. Plasma Phys., accepted. 